\title{
Synthesis of hexahydroquinolines using the new ionic liquid sulfonic acid functionalized pyridinium chloride as a catalyst
}

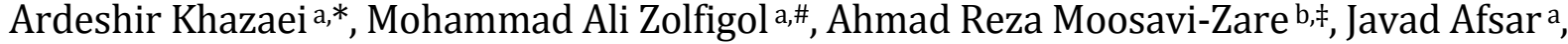 \\ Abdolkarim Zare c, Vahid Khakyzadeh a, Mohammad Hassan Beyzavi ${ }^{d}$ \\ a Faculty of Chemistry, Bu-Ali Sina University, Hamedan, 6517838683, Iran \\ b Department of Chemistry, University of Sayyed Jamaleddin Asadabadi, Asadabad, 6541835583, Iran \\ c Department of Chemistry, Payame Noor University, PO BOX 19395-4697 Tehran, Iran \\ d Institut für Chemie und Biochemie, Freie Universität Berlin, Takustr. 3, 14195 Berlin, Germany
}

\section{A R T I C L E I N F O}

\section{Article history:}

Received 31 May 2013

Accepted 14 August 2013

Published 20 October 2013

Keywords:

Brönsted acidic ionic liquid

Sulfonic acid functionalized pyridinium

chloride $\left[\right.$ Pyridine- $\left.\mathrm{SO}_{3} \mathrm{H}\right] \mathrm{Cl}$

Hexahydroquinoline

Solvent-free synthesis

\section{A B S T R A C T}

Sulfonic acid functionalized pyridinium chloride [pyridine- $\left.\mathrm{SO}_{3} \mathrm{H}\right] \mathrm{Cl}$ has been synthesized as a novel Brönsted acidic ionic liquid and characterized on the basis of its FT-IR, ${ }^{1} \mathrm{H}$ and ${ }^{13} \mathrm{C}$ NMR, MS, thermogravimetry, and derivative thermogravimetry data. The material has also been used as a highly efficient, homogeneous, and reusable catalyst for the preparation of hexahydroquinolines according to the one-pot multi-component condensation of arylaldehydes, dimedone (5,5-dimethylcyclohexane-1,3-dione), $\beta$-ketoesters, and ammonium acetate under solvent-free conditions.

(C) 2013, Dalian Institute of Chemical Physics, Chinese Academy of Sciences. Published by Elsevier B.V. All rights reserved.

\section{Introduction}

Ionic liquids (ILs) have attracted considerable interest from chemists during the course of the last two decades because of their unique properties, including their non-flammability, non-volatility, wide liquid-state temperature range, high thermal and chemical stability, large electrochemical window, and favorable solvation behaviors [1,2]. These compounds have been applied extensively to a number of different areas, including spectroscopy, extraction, separation, and electrochemistry processes $[1,3]$ and are used as solvents, catalysts, and reagents in a variety of organic transformations [1,2,4-10]. Of the many different kinds of ILs available, the Brönsted acidic ILs offer the greatest potential for the development of envi- ronmentally friendly acid catalysts for organic synthesis because they effectively combine the advantages of liquid and solid acids and provide high levels of operational simplicity, efficacy, and selectivity coupled with a low environmental impact [11-18].

Multi-component reactions play an important role in combinatorial chemistry because they allow for complex target compounds to be synthesized in a single step from three or more reactants with greater simplicity, efficiency, and atom economy than the corresponding conventional chemical approaches [5,19-21].

Some pharmaceutical agents can be photolabile, and the photochemical decomposition of such materials following their exposure to light could result in the reduction or complete loss

\footnotetext{
* Corresponding author. Tel: +98-8118282807; Fax: +98-8118257407; E-mail: Khazaei_1326@yahoo.com

\# Corresponding author. Tel: +98-8118282807; Fax: +98-8118257407; E-mail: mzolfigol@yahoo.com

‡Corresponding author. Tel: +98-8123237450; Fax: +98-8123237451; E-mail: moosavizare@yahoo.com

DOI: 10.1016/S1872-2067(12)60678-0 | http://www.sciencedirect.com/science/journal/18722067 | Chin. J. Catal., Vol. 34, No. 10, October 2013
} 
of their desired pharmacological activity. Furthermore, photochemical decomposition could even lead to changes in their properties that could turn them into potentially toxic compounds. In some cases, the products resulting from photochemical decomposition could induce hypersensitivity to light and potentially give rise to photoallergic and phototoxic effects $[22,23]$. For example, 1,4-dihydropyridine (DHP) derivatives are included in the long list of photosensitive pharmaceuticals [24,25].

According to research available in the literature, one approach for reducing the photosensitivity of pharmaceutical agents is to modify their structure and their core scaffold. Modifications to the DHP ring, such as the introduction of hetero atoms or different substituents, could not only result in decreased photosensitivity in these compounds but could also enrich the diversity of DHP-based chemistry and provide structure-activity relationships that would provide novel insights into the molecular interactions of these compounds with their target [26,27]. It is also recognized that structural modifications to the DHP periphery could have a significant impact on their pharmacological activity [28-30]. One series of DHP derivatives with an improved structural scaffold, the hexahydroquinoline derivatives (HHQs), can be synthesized according to a Hantzsch synthesis using aryl aldehydes, dimedone (5,5-dimethylcyclohexane-1,3-dione), $\beta$-ketoesters, and ammonium acetate in a one-pot multi-component condensation reaction $[31,32]$. Quinolines bearing a 1,4-dihydropyridine scaffold have been identified as promising structure in medicinal chemistry because of their many pharmacological properties, including their antibacterial antihypertensive, anti-inflammatory, antimalarial, antiasthmatic, and tyrosine kinase inhibitory activity [33-38]. Furthermore, various methods and catalysts have been developed for the preparation of HHQs [39-47].

Herein, we report for the first time the synthesis of sulfonic acid functionalized pyridinium chloride [Pyridine- $\left.\mathrm{SO}_{3} \mathrm{H}\right] \mathrm{Cl}$ as a new Brönsted acidic ionic liquid from inexpensive and commercially available starting materials (Scheme 1), and its full characterization using FT-IR, ${ }^{1} \mathrm{H}$ and ${ }^{13} \mathrm{C}$ NMR, thermal gravimetric analysis (TGA) and derivative thermal gravimetric analysis (DTGA). Furthermore, we report the use of this ionic liquid as a highly efficient, homogeneous, and recyclable catalyst for

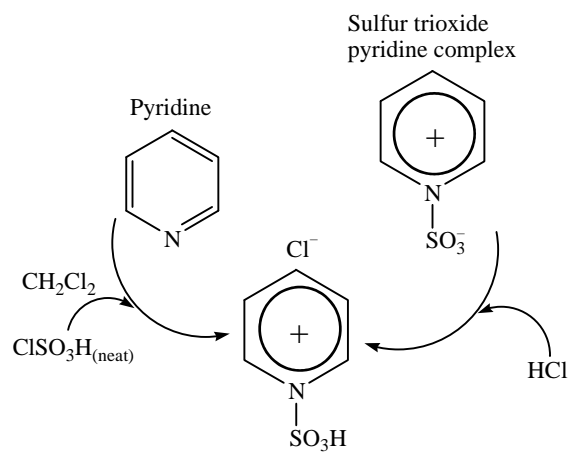

Scheme 1. Preparation of sulfonic acid functionalized pyridinium chloride [pyridine- $\mathrm{SO}_{3} \mathrm{H}$ ]Cl. Left: The reaction of pyridine (1 equiv.) with chlorosulfonic acid ( 1 equiv.) in dry $\mathrm{CH}_{2} \mathrm{Cl}_{2}$ at $0{ }^{\circ} \mathrm{C}$; Right: The reaction of sulfur trioxide pyridine complex with an excess of $\mathrm{HCl}$ gas at room temperature. the preparation of hexahydroquinolines in the one-pot multi-component condensation of dimedone, arylaldehydes, $\beta$-ketoesters, and ammonium acetate under solvent-free conditions (Scheme 2).

\section{Experimental}

All of the chemicals used in the current study were purchased from Merck or Fluka Chemical. The known products were identified by comparison of their melting points and spectral data with those reported in the literature. The chemical reactions were monitored by TLC using silica gel SIL G/UV 254 plates. ${ }^{1} \mathrm{H}$ NMR (400 or $300 \mathrm{MHz}$ ) and ${ }^{13} \mathrm{C}$ NMR (100 or 75 $\mathrm{MHz}$ ) analyses were run on a Bruker Avance DPX-250 FT-NMR spectrometer. The chemical shifts $(\delta)$ have been reported relative to TMS, which was used as an internal reference. Melting points were recorded on a Büchi B-545 apparatus in open capillary tubes.

\subsection{General procedure for the preparation of the [pyridine-SO $3 \mathrm{H}$ ] Cl ionic liquid}

A solution of pyridine $(0.36 \mathrm{~g}, 5 \mathrm{mmol})$ in $\mathrm{CH}_{2} \mathrm{Cl}_{2}(40 \mathrm{~mL})$ was added drop-wise to a stirred solution of chlorosulfonic acid $(0.58 \mathrm{~g}, 5 \mathrm{mmol})$ in dry $\mathrm{CH}_{2} \mathrm{Cl}_{2}(40 \mathrm{~mL})$ at $0{ }^{\circ} \mathrm{C}$ over a period of $10 \mathrm{~min}$, and the resulting mixture was stirred for $20 \mathrm{~min}$ before being allowed to stand for $5 \mathrm{~min}$. The $\mathrm{CH}_{2} \mathrm{Cl}_{2}$ layer was then decanted and the resulting residue was triturated with $\mathrm{CH}_{2} \mathrm{Cl}_{2}$ $(3 \times 10 \mathrm{~mL})$ before being dried under vacuum at $90{ }^{\circ} \mathrm{C}$ to give [pyridine- $\mathrm{SO}_{3} \mathrm{H}$ ] Cl as a viscous colorless oil in $95 \%$ yield $(0.93$ g).

Spectral data of [pyridine- $\mathrm{SO}_{3} \mathrm{H}$ ]Cl are as follows. Viscous colorless oil; IR (Nujol): 750, 866, 1042, 1174, 1488, 2650-3550 cm-1; ${ }^{1} \mathrm{H}$ NMR (300 MHz, DMSO-d 6 ): $\delta 8.11(\mathrm{t}, J=$ $7.45 \mathrm{~Hz}, 2 \mathrm{H}), 8.65(\mathrm{t}, J=7.81 \mathrm{~Hz}, 1 \mathrm{H}), 8.94(\mathrm{~d}, J=5.76 \mathrm{~Hz}, 2 \mathrm{H})$, 13.67 (s, 1H); ${ }^{13} \mathrm{C}$ NMR (75 MHz, DMSO-d6): $\delta 127.9,142.4$, 147.1; MS: $m / z=196[\mathrm{M}+\mathrm{H}]^{+}, 195[\mathrm{M}]^{+}$.

\subsection{General procedure for the synthesis of hexahydroquinolines}

[Pyridine-SO ${ }_{3} \mathrm{H}$ ] Cl (11.7 mg, $\left.0.06 \mathrm{mmol}, 3 \mathrm{~mol} \%\right)$ was added to a mixture of dimedone $(0.28 \mathrm{~g}, 2 \mathrm{mmol})$, arylaldehyde (2 mmol), $\beta$-ketoester $(2 \mathrm{mmol})$, and ammonium acetate $(0.185 \mathrm{~g}$, $2.4 \mathrm{mmol}$ ) in a test tube, and the resulting mixture was initially stirred magnetically before being agitated with a small rod at $50{ }^{\circ} \mathrm{C}$ following solidification of the reaction mixture. Upon

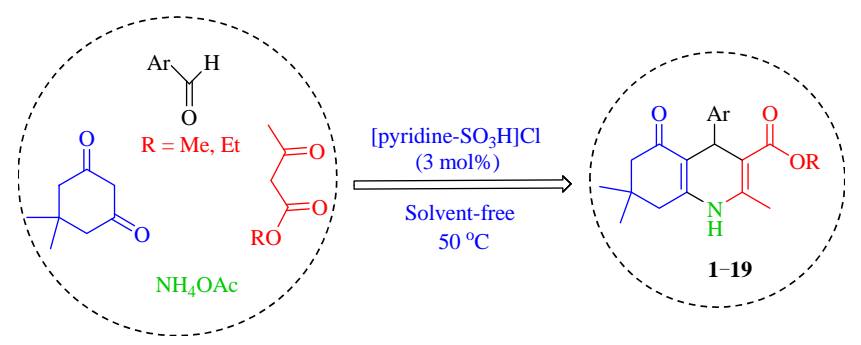

Scheme 2. One-pot multi-component preparation of hexahydroquinolines catalyzed by [pyridine- $\left.\mathrm{SO}_{3} \mathrm{H}\right] \mathrm{Cl}$. 
completion of the reaction, as determined by TLC, the reaction mixture was cooled to room temperature and diluted with ethyl acetate $(20 \mathrm{~mL})$. The resulting mixture was then stirred at reflux for $3 \mathrm{~min}$ and the solvent decanted (the product is soluble in hot ethyl acetate; however, [pyridine- $\mathrm{SO}_{3} \mathrm{H}$ ] Cl is not soluble in this solvent). The remaining viscous residue (oil) was washed with hot ethyl acetate $(10 \mathrm{~mL})$ to give the pure recycled catalyst. The decanted solutions were then combined, washed with water $(20 \mathrm{~mL})$, and dried. The solvent was then evaporated to give the crude product, which was purified by recrystallization from ethanol (95\%) or column chromatography using $n$-hexane/ethyl acetate $(4 / 1, v / v)$ as an eluent. In the current work, [Pyridine- $\left.\mathrm{SO}_{3} \mathrm{H}\right] \mathrm{Cl}$ was recycled and reused four times without significant loss of its catalytic activity.

Ethyl 2,7,7-trimethyl-5-oxo-4-phenyl-1,4,5,6,7,8-hexahydroquinoline-3-carboxylate (1). White solid; isolated yield $94 \%$ (0.32 g); melting point: 206-208 ${ }^{\circ} \mathrm{C}$ (Ref. [30]: 203-205 ${ }^{\circ} \mathrm{C}$ ); ${ }^{1} \mathrm{H}$ NMR (300 MHz, DMSO-d6): $\delta 0.85(\mathrm{~s}, 3 \mathrm{H}), 1.00(\mathrm{~s}, 3 \mathrm{H}), 1.13(\mathrm{t}, J$ $=7.0 \mathrm{~Hz}, 3 \mathrm{H}), 2.01-2.20(\mathrm{~m}, 2 \mathrm{H}), 2.29(\mathrm{~s}, 3 \mathrm{H}), 2.38-2.50(\mathrm{~m}$, $2 \mathrm{H}), 3.97(\mathrm{q}, J=7.0 \mathrm{~Hz}, 2 \mathrm{H}), 4.82(\mathrm{~s}, 1 \mathrm{H}), 7.05(\mathrm{~m}, 1 \mathrm{H}), 7.18(\mathrm{t}, J$ $=6.7 \mathrm{~Hz}, 2 \mathrm{H}), 7.21(\mathrm{t}, J=6.5 \mathrm{~Hz}, 2 \mathrm{H}), 9.12(\mathrm{~s}, 1 \mathrm{H}) ;{ }^{13} \mathrm{C}$ NMR $(75$ MHz, DMSO-d6): $\delta 14.5,18.8,26.8,29.5,32.6,36.5,50.6,59.6$, $103.4,109.9,113.5,126.9,128.8,130.5,146.0,150.3,167.0$, 194.7.

Ethyl 2,7,7-trimethyl-4-(4-nitrophenyl)-5-oxo-1,4,5,6,7,8hexahydroquinoline-3-carboxylate (2). White solid; isolated yield 95\% (0.37 g); melting point: $245-248{ }^{\circ} \mathrm{C}$ (Ref. [34]: 242-244 ${ }^{\circ} \mathrm{C}$ ); ${ }^{1} \mathrm{H}$ NMR (500 MHz, $\left.\mathrm{CDCl}_{3}\right): \delta 0.92(\mathrm{~s}, 3 \mathrm{H}), 1.10(\mathrm{~s}$, $3 \mathrm{H}), 1.19(\mathrm{t}, J=7.1 \mathrm{~Hz}, 3 \mathrm{H}), 2.16(\mathrm{~d}, J=16.4 \mathrm{~Hz}, 2 \mathrm{H}), 2.24-2.29$ (distorted AB system, 2H), $2.41(\mathrm{~s}, 3 \mathrm{H}), 4.07$ (q, $J=7.1 \mathrm{~Hz}, 2 \mathrm{H}$ ), $5.18(\mathrm{~s}, 1 \mathrm{H}), 6.68(\mathrm{~s}, 1 \mathrm{H}), 7.51(\mathrm{~d}, J=8.5 \mathrm{~Hz}, 2 \mathrm{H}), 8.09$ (d, $J=8.5$ $\mathrm{Hz}, 2 \mathrm{H}) ;{ }^{13} \mathrm{C} \mathrm{NMR}\left(125 \mathrm{MHz}, \mathrm{CDCl}_{3}\right): \delta 14.6,19.8,27.5,29.8$, $33.1,37.7,41.3,51.0,60.5,105.3,111.4,123.7,129.4,145.0$, 146.6, 149.6, 154.9, 167.3, 195.9.

Ethyl 2,7,7-trimethyl-4-(3-nitrophenyl)-5-oxo-1,4,5,6,7,8hexahydroquinoline-3-carboxylate (3). White solid; isolated yield 94\% (0.36 g); melting point: $179-181{ }^{\circ} \mathrm{C}$ (Ref. [32]: $\left.177-178^{\circ} \mathrm{C}\right)$; IR (KBr): 3285, 2959, 1705, 1606, 1487, 1380, $1212 \mathrm{~cm}^{-1}$; ${ }^{1} \mathrm{H}$ NMR (400 MHz, DMSO-d $): \delta(\mathrm{s}, 3 \mathrm{H}), 1.00(\mathrm{~s}, 3 \mathrm{H})$, $1.10(\mathrm{t}, J=7.04 \mathrm{~Hz}, 3 \mathrm{H}), 1.98(\mathrm{~d}, J=16.12 \mathrm{~Hz}, 1 \mathrm{H}), 2.19(\mathrm{~d}, J=$ $16.12 \mathrm{~Hz}, 1 \mathrm{H}$ ), $2.31(\mathrm{~s}, 3 \mathrm{H}), 2.42-2.50$ (distorted $\mathrm{AB}$ system, 2H), 3.99-3.94 (m, 2H), $4.95(\mathrm{~s}, 1 \mathrm{H}), 7.52(\mathrm{t}, J=8.0 \mathrm{~Hz}, 1 \mathrm{H}), 7.61$ (d, $7.68 \mathrm{~Hz}, 1 \mathrm{H}), 7.97$ (t, $J=2.16 \mathrm{~Hz}, 2 \mathrm{H}), 9.25(\mathrm{~s}, 1 \mathrm{H}) ;{ }^{13} \mathrm{C} \mathrm{NMR}$ (400 MHz, DMSO-d6): $\delta 13.99,18.31,26.30,29.01,32.16,36.36$, $38.8,49.98,59.22,102.59,109.17,120.89,121.95,129.41$, 134.28, 146.11, 147.34, 149.69, 150.12, 166.36, 194.31 .

Ethyl 4-(4-methoxyphenyl)-2,7,7-trimethyl-5-oxo-1,4,5,6,7, 8-hexahydroquinoline-3-carboxylate (4). White solid; isolated yield 95\% (0.35 g); melting point: $256-258{ }^{\circ} \mathrm{C}$ (Ref. [33]: 257-259 ${ }^{\circ} \mathrm{C}$ ); ${ }^{1} \mathrm{H}$ NMR (300 MHz, DMSO-d 6 ): $\delta 0.85$ (s, 3H), 1.00 $(\mathrm{s}, 3 \mathrm{H}), 1.14$ (t, J=7.0 Hz, 3H), 1.96 (d, J=16.0 Hz, 1H), 2.15 (d, J $=16.1 \mathrm{~Hz}, 1 \mathrm{H}), 2.27(\mathrm{~s}, 3 \mathrm{H}), 2.37-2.49(\mathrm{~m}, 2 \mathrm{H}), 3.66(\mathrm{~s}, 3 \mathrm{H})$, 3.97 (q, J = 7.0 Hz, 2H), 4.79 (s, 1H), 6.73 (d, J=8.3 Hz, 2H), 7.05 (d, $J=8.3 \mathrm{~Hz}, 2 \mathrm{H}), 8.99$ (s, 1H); ${ }^{13} \mathrm{C}$ NMR (75 MHz, DMSO-d 6$): \delta$ 14.6, 18.7, 26.9, 29.6, 32.6, 35.4, 50.6, 55.3, 59.4, 104.4, 110.7, $113.5,128.8,140.5,145.1,149.7,157.7,167.4,194.7$.

Ethyl 2,7,7-trimethyl-5-oxo-4-p-tolyl-1,4,5,6,7,8-hexahy- droquinoline-3-carboxylate (5). White solid; isolated yield $92 \%$ (0.32 g); melting point: $259-262{ }^{\circ} \mathrm{C}$ (Ref. [33]: $260-261{ }^{\circ} \mathrm{C}$ ); ${ }^{1} \mathrm{H}$ NMR (500 MHz, $\left.\mathrm{CDCl}_{3}\right): \delta 0.96(\mathrm{~s}, 3 \mathrm{H}), 1.08(\mathrm{~s}, 3 \mathrm{H}), 1.24(\mathrm{t}, J=$ $7.1 \mathrm{~Hz}, 3 \mathrm{H}), 2.15-2.31(\mathrm{~m}, 7 \mathrm{H}), 2.35(\mathrm{~s}, 3 \mathrm{H}), 4.09$ (q, $J=7.1 \mathrm{~Hz}$, 2H), $5.04(\mathrm{~s}, 1 \mathrm{H}), 6.76(\mathrm{~s}, 1 \mathrm{H}), 7.02(\mathrm{~d}, J=7.8 \mathrm{~Hz}, 2 \mathrm{H}), 7.21(\mathrm{~d}, J=$ $7.8 \mathrm{~Hz}, 2 \mathrm{H}) ;{ }^{13} \mathrm{C}$ NMR $\left(125 \mathrm{MHz}, \mathrm{CDCl}_{3}\right): \delta 14.7,19.7,21.5,27.6$, 29.8, 33.1, 36.6, 41.3, 51.2, 60.2, 106.6, 112.4, 128.3, 129.0, 135.8, 143.9, 144.7, 149.3, 167.9, 196.1.

Ethyl 4-(4-hydroxyphenyl)-2,7,7-trimethyl-5-oxo-1,4,5,6,7, 8-hexahydroquinoline-3-carboxylate (6). White solid; isolated yield $94 \%$ (0.33 g); melting point: $234-236{ }^{\circ} \mathrm{C}$ (Ref. [33]: 232-234 ${ }^{\circ} \mathrm{C}$ ); ${ }^{1} \mathrm{H}$ NMR (300 MHz, DMSO-d6): $\delta 0.86(\mathrm{~s}, 3 \mathrm{H}), 1.00$ $(\mathrm{s}, 3 \mathrm{H}), 1.14(\mathrm{t}, J=7.0 \mathrm{~Hz}, 3 \mathrm{H}), 1.96(\mathrm{~d}, J=16.0 \mathrm{~Hz}, 1 \mathrm{H}), 2.15(\mathrm{~d}, J$ $=16.1 \mathrm{~Hz}, 1 \mathrm{H}), 2.26(\mathrm{~s}, 3 \mathrm{H}), 2.36-2.49(\mathrm{~m}, 2 \mathrm{H}), 3.96(\mathrm{q}, J=7.0$ $\mathrm{Hz}, 2 \mathrm{H}), 4.74(\mathrm{~s}, 1 \mathrm{H}), 6.56(\mathrm{~d}, J=8.1 \mathrm{~Hz}, 2 \mathrm{H}), 6.93$ (d, $J=8.1 \mathrm{~Hz}$, 2H), 8.94 (s, 1H), 9.01 (s, 1H); ${ }^{13} \mathrm{C}$ NMR (75 MHz, DMSO-d 6$): \delta$ 14.6, 18.7, 26.9, 29.6, 32.6, 35.3, 50.8, 59.4, 104.6, 110.8, 114.9, 128.8, 138.9, 144.8, 149.6, 155.7, 167.5, 194.7.

Ethyl 4-(4-bromophenyl)-2,7,7-trimethyl-5-oxo-1,4,5,6,7,8hexahydroquinoline-3-carboxylate (7). White solid; isolated yield $91 \%$ (0.38 g); melting point: $252-254{ }^{\circ} \mathrm{C}$ (Ref. [30]:

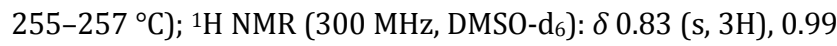
$(\mathrm{s}, 3 \mathrm{H}), 1.10(\mathrm{t}, J=6.9 \mathrm{~Hz}, 3 \mathrm{H}), 1.96(\mathrm{~d}, J=16.0 \mathrm{~Hz}, 1 \mathrm{H}), 2.16(\mathrm{~d}, J$ $=16.1 \mathrm{~Hz}, 1 \mathrm{H}), 2.29(\mathrm{~s}, 3 \mathrm{H}), 2.38-2.49(\mathrm{~m}, 2 \mathrm{H}), 3.97(\mathrm{q}, J=7.0$ $\mathrm{Hz}, 2 \mathrm{H}), 4.84(\mathrm{~s}, 1 \mathrm{H}), 7.11(\mathrm{~d}, J=7.2 \mathrm{~Hz}, 2 \mathrm{H}), 7.37$ (d, $J=7.2 \mathrm{~Hz}$, 2H), 9.09 (s, $1 \mathrm{H}) ;{ }^{13} \mathrm{C}$ NMR (75 MHz, DMSO-d 6$): \delta 14.6,18.8$, 26.9, 29.5, 32.6, 36.2, 50.6, 59.5, 103.5, 110.1, 119.1, 130.2, 131.0, 145.8, 147.4, 150.0, 167.1, 194.7.

Ethyl 4-(4-chlorophenyl)-2,7,7-trimethyl-5-oxo-1,4,5,6,7,8hexahydroquinoline-3-carboxylate (9). White solid; isolated yield 91\% (0.35 g); melting point: $244-246{ }^{\circ} \mathrm{C}$ (Ref. [32]: 245-246 ${ }^{\circ} \mathrm{C}$ ); IR (KBr): 3274, 1707, 1605, 1489, $1382 \mathrm{~cm}^{-1} ;{ }^{1} \mathrm{H}$ NMR (400 MHZ, DMSO-d6): $\delta 0.82(\mathrm{~s}, 3 \mathrm{H}), 0.99(\mathrm{~s}, 3 \mathrm{H}), 1.11(\mathrm{t}, J$ $=7.08 \mathrm{~Hz}, 3 \mathrm{H}), 1.97(\mathrm{~d}, J=16.08,3 \mathrm{H}), 2.16(\mathrm{~d}, J=16.12 \mathrm{~Hz}, 1 \mathrm{H})$, 2.43-2.50 (distorted AB system, $2 \mathrm{H}$ ), $3.96(\mathrm{~d}, J=7.08 \mathrm{~Hz}, 2 \mathrm{H}$ ), 4.83 (s, 1H), 7.15 (d, J = 8.48, 2H), 7.24 (d, J=8.44, 2H), 9.01 (s, $1 \mathrm{H})$.

Ethyl 4-(2-methoxyphenyl)-2,7,7-trimethyl-5-oxo-1,4,5,6,7, 8-hexahydroquinoline-3-carboxylate (10). White solid; isolated yield 96\% (0.35 g); melting point:257-259 ${ }^{\circ} \mathrm{C}$ (Ref. [10]: 256-257 ${ }^{\circ} \mathrm{C}$ ); IR (KBr): 3285, 2957, 1689, 1611, 1488, 1381, $1216 \mathrm{~cm}^{-1}$; ${ }^{1} \mathrm{H}$ NMR (400 MHz, DMSO-d $): \delta 0.83$ (s, 3H), 0.99 (s, $3 \mathrm{H}), 1.10(\mathrm{t}, J=7.08 \mathrm{~Hz}, 3 \mathrm{H}), 1.89(\mathrm{~d}, J=16.08 \mathrm{~Hz}, 1 \mathrm{H}), 2.11(\mathrm{~d}, J$ $=16.08 \mathrm{~Hz}, 1 \mathrm{H}), 2.18(\mathrm{~s}, 3 \mathrm{H}), 2.40-2.50$ (distorted $\mathrm{AB}$ system, 2H), $3.68(\mathrm{~s}, 3 \mathrm{H}), 3.91(\mathrm{q}, J=3.64 \mathrm{~Hz}, 2 \mathrm{H}), 5.04(\mathrm{~s}, 1 \mathrm{H}), 6.75(\mathrm{~d}, J$ $=7.44 \mathrm{~Hz}, 1 \mathrm{H}), 6.82(\mathrm{~d}, J=8.04 \mathrm{~Hz}, 1 \mathrm{H}), 7.03(\mathrm{~d}, J=7.36 \mathrm{~Hz}, 1 \mathrm{H})$, 7.09 (q, $J=1.44 \mathrm{~Hz}, 1 \mathrm{H}), 8.93(\mathrm{~s}, 1 \mathrm{H}) ;{ }^{13} \mathrm{C}$ NMR $(400 \mathrm{MHz}$, DMSO-d6): $\delta$ 14.05, 18.00, 26.15, 29.28, 31.95, 32.77, 50.36, 55.14, 58.72, 102.89, 108.64, 110.96, 119.44, 126.90, 130.46, $134.93,144.08,149.94,157.10,167.26,193.79$.

Ethyl 4-(2,4-dichlorophenyl)-2,7,7-trimethyl-5-oxo-1,4,5,6, 7,8-hexahydroquinoline-3-carboxylate (11). White solid; isolated yield $94 \%$ ( $0.38 \mathrm{~g}$ ); melting point: $243-245{ }^{\circ} \mathrm{C}$; IR (KBr): 3285, 1706, 1609, 1493, 1381, $1214 \mathrm{~cm}^{-1}$; ${ }^{1} \mathrm{H}$ NMR (400 MHz, DMSO-d $): \delta 0.83(\mathrm{~s}, 3 \mathrm{H}), 0.92(\mathrm{~s}, 3 \mathrm{H}), 1.07(\mathrm{t}, J=7.08 \mathrm{~Hz}, 3 \mathrm{H})$, $1.91(\mathrm{~d}, J=16.04 \mathrm{~Hz}, 1 \mathrm{H}), 2.14(\mathrm{~d}, J=16.08 \mathrm{~Hz}, 1 \mathrm{H}), 2.22(\mathrm{~s}, 3 \mathrm{H})$, 2.49-2.50 (distorted AB system, 2H), 3.95-3.91 (m, 2H), 5.14 (s, 
1H), $7.31(\mathrm{~s}, 2 \mathrm{H}), 7.34(\mathrm{~s}, 1 \mathrm{H}), 9.12(\mathrm{~s}, 1 \mathrm{H}) ;{ }^{13} \mathrm{C} \mathrm{NMR}(400 \mathrm{MHz}$, DMSO-d 6 ): $\delta 14.07,18.19,26.37,29.01,31.94,34.73,50.14$, 58.99, 102.69, 109.18, 126.83, 128.16, 130.70, 132.75, 132.82,144.19, 145.44, 149.85, 196.3; MS: $m / z=373$ [M]+; CHN Analysis: Anal. Calcd for $\mathrm{C}_{21} \mathrm{H}_{23} \mathrm{Cl}_{2} \mathrm{NO}_{3}$ : C 61.77, H 5.68, N 3.43; Found C 61.43, H 5.96, N 3.27.

Ethyl 2,7,7-trimethyl-5-oxo-4-(naphthalen-2-yl)-1,4,5,6,7,8hexahydroquinoline-3-carboxylate (12). White solid; isolated yield $92 \%$ (0.36 g); melting point: $235-237{ }^{\circ} \mathrm{C}$; IR (KBr): 3253 , 1701, 1607, 1493, $1217 \mathrm{~cm}^{-1} ;{ }^{1} \mathrm{H}$ NMR (400 MHz, DMSO-d 6 ): $\delta$ $0.82(\mathrm{~s}, 3 \mathrm{H}), 1.00(\mathrm{~s}, 3 \mathrm{H}), 1.12(\mathrm{t}, J=7.08 \mathrm{~Hz}, 3 \mathrm{H}), 1.95(\mathrm{~d}, J=$ $16.12 \mathrm{~Hz}, 1 \mathrm{H}$ ), 2.18 (d, $J=16.16 \mathrm{~Hz}, 1 \mathrm{H}), 2.31$ (s, 3H), 2.42-2.50 (distorted AB system, 2H), $3.95(\mathrm{q}, J=7.12 \mathrm{~Hz}, 2 \mathrm{H}), 5.03(\mathrm{~s}, 1 \mathrm{H})$, 7.43-7.36 (m, 3H), 7.58 (s, 1H), 7.79-7.78 (m, 3H), $9.11(\mathrm{~s}, 1 \mathrm{H})$,; ${ }^{13} \mathrm{C}$ NMR (400 MHz, DMSO-d6): $\delta$ 14.12, 18.29, 26.35, 29.12, $32.10,36.20,50.21,59.02,103.38,109.74,125.11,125.32$, $125.73,126.68,127.22,127.28,127.56,131.60,132.72,144.99$, 145.14, 149.64, 166.81, 194.30; MS: $m / z=389[\mathrm{M}]+$; CHN Analysis: Anal. Calcd for $\mathrm{C}_{25} \mathrm{H}_{27} \mathrm{NO}_{3}$ : C 77.09, H 6.99, N 3.60; Found C 76.88, H 6.91, N 3.68.

Ethyl 4-(3-hydroxyphenyl)-2,7,7-trimethyl-5-oxo-1,4,5,6,7, 8-hexahydroquinoline-3-carboxylate (13). White solid; isolated yield $92 \%$ (0.33 g); melting point: $211-213{ }^{\circ} \mathrm{C}$; IR (KBr): 3408 , 3289, 1675, 1606, 1485, $1220 \mathrm{~cm}^{-1} ;{ }^{1} \mathrm{H}$ NMR $(400 \mathrm{MHz}$, DMSO-d 6 ): $\delta 0.86(\mathrm{~s}, 3 \mathrm{H}), 1.00(\mathrm{~s}, 3 \mathrm{H}) .1 .13(\mathrm{t}, J=7.04 \mathrm{~Hz}, 3 \mathrm{H})$, $2.00(\mathrm{~s}, 1 \mathrm{H}), 2.15(\mathrm{~d}, J=16.08 \mathrm{~Hz}, 1 \mathrm{H}), 2.26(\mathrm{~s}, 3 \mathrm{H}), 2.38-2.50$ (distorted AB system, 2H), $3.97(\mathrm{q}, J=7.08 \mathrm{~Hz}, 2 \mathrm{H}), 4.78(\mathrm{~s}, 1 \mathrm{H})$, 6.46-6.43 (m, $1 \mathrm{H}), 6.57$ (d, $J=7.24 \mathrm{~Hz}, 2 \mathrm{H}), 6.93(\mathrm{t}, J=7.96 \mathrm{~Hz}$, 1H), $9.01(\mathrm{~s}, 1 \mathrm{H}), 9.06(\mathrm{~s}, 1 \mathrm{H}) ;{ }^{13} \mathrm{C}$ NMR (22.5 MHz, DMSO-d 6$): \delta$ 14.8, 18.9, 27.4, 29.8, 32.8, 36.4, 40.4, 51.2, 59.7, 104.6, 110.8, 113.4, 115.4, 119.0, 129.3, 145.4, 150.2, 157.7, 167.8, 195.0; MS: $m / z=355[\mathrm{M}]^{+}$; CHN Analysis: Anal. Calcd for $\mathrm{C}_{21} \mathrm{H}_{25} \mathrm{NO}_{4}: \mathrm{C}$ 70.96, H 7.09, N 3.94; Found C 70.67, H 7.18, N 3.81.

Methyl 4-(4-methoxyphenyl)-2,7,7-trimethyl-5-oxo-1,4,5,6, 7,8-hexahydroquinoline-3-carboxylate (17). White solid; isolated yield $91 \%$ ( $0.32 \mathrm{~g}$ ); melting point: $257-259{ }^{\circ} \mathrm{C}$ (Ref. [10]: 256-257 ${ }^{\circ} \mathrm{C}$ ); ${ }^{1} \mathrm{H}$ NMR (300 MHz, DMSO-d 6 ): $\delta 0.84(\mathrm{~s}, 3 \mathrm{H}), 0.99$ (s, 3H), 1.97 (d, J = $16.0 \mathrm{~Hz}, 1 \mathrm{H}), 2.15$ (d, $J=16.1 \mathrm{~Hz}, 1 \mathrm{H}), 2.28$ (s, 3H), 2.37-2.49 (m, 2H), $3.52(\mathrm{~s}, 3 \mathrm{H}), 3.66(\mathrm{~s}, 3 \mathrm{H}), 4.81(\mathrm{~s}$, $1 \mathrm{H}), 6.73(\mathrm{~d}, J=7.4 \mathrm{~Hz}, 2 \mathrm{H}), 7.05(\mathrm{~d}, J=7.4 \mathrm{~Hz}, 2 \mathrm{H}), 9.02(\mathrm{~s}, 1 \mathrm{H})$; ${ }^{13} \mathrm{C}$ NMR (75 MHz, DMSO-d 6 ): $\delta 18.7,26.9,29.6,32.6,35.2,50.7$, 51.1, 55.3, 104.0, 110.7, 113.6, 128.7, 140.3, 145.4, 149.7, 157.7, 167.9, 194.7.

Methyl 4-(3-bromophenyl)-2,7,7-trimethyl-5-oxo-1,4,5,6,7, 8-hexahydroquinoline-3-carboxylate (18). White solid; isolated yield $90 \%$ (0.36 g); melting point: $233-234{ }^{\circ} \mathrm{C}$ (Ref. [10]: 235-236 ${ }^{\circ} \mathrm{C}$ ); ${ }^{1} \mathrm{H}$ NMR (300 MHz, DMSO-d 6 ): $\delta 0.83$ (s, 3H), 1.00 (s, 3H), 1.99 (d, $J=16.0 \mathrm{~Hz}, 1 \mathrm{H}), 2.18$ (d, $J=16.1 \mathrm{~Hz}, 1 \mathrm{H}), 2.30$ (s, 3H), 2.39-2.49 (m, 2H), $3.53(\mathrm{~s}, 3 \mathrm{H}), 4.85$ (s, 1H), 7.14-7.16 (m, 2H), 7.25-7.27 (m, 2H), $9.15(\mathrm{~s}, 1 \mathrm{H}) ;{ }^{13} \mathrm{C}$ NMR $(75 \mathrm{MHz}$, DMSO-d6): $\delta$ 18.8, 26.8, 29.5, 32.6, 36.3, 103.1, 109.9, 121.6, 126.8, 129.1, 130.5, 130.6, 146.3, 150.3, 150.5, 167.5, 194.7; MS: $m / z=404[\mathrm{M}]+$; CHN Analysis: Anal. Calcd for $\mathrm{C}_{20} \mathrm{H}_{22} \mathrm{BrNO}_{3}$ : C 59.42, H 5.48, N 3.46; Found C 59.61, H 5.27, N 3.39.

Diethyl 4,4'-(1,4-phenylene)bis(2,7,7-trimethyl-5-oxo-1,4,5, 6,7,8-hexahydroquinoline-3-carboxylate) (19). White solid; isolated yield $76 \%$ (0.46 g); melting point: $294-296{ }^{\circ} \mathrm{C}$; IR
(KBr): 3284, 3215, 2961, 1702, 1612, 1493, 1379, $1218 \mathrm{~cm}^{-1}$; 1H NMR (400 MHz, DMSO-d 6 ): $\delta 0.97(\mathrm{~s}, 12 \mathrm{H}), 1.06(\mathrm{t}, J=6.96$ $\mathrm{Hz}, 6 \mathrm{H}), 2.09-2.13(\mathrm{~m}, 2 \mathrm{H}), 2.26$ (s, 6H) 2.45-2.50 (m, 2H), 3.96 (q, $J=3.96 \mathrm{~Hz}, 4 \mathrm{H}), 4.79(\mathrm{~s}, 2 \mathrm{H}), 6.92(\mathrm{~s}, 4 \mathrm{H}), 9.01(\mathrm{~s}, 2 \mathrm{H}) ;{ }^{13} \mathrm{C}$ NMR (400 MHz, DMSO-d $): \delta 14.08,18.28,26.75,27.91,28.85$, 32.13, 34.77, 50.22, 58.97, 102.38, 103.35, 109.82, 126.61, 144.65, 149.61, 166.90; MS: $m / z=600[\mathrm{M}]^{+}$; CHN Analysis: Anal. Calcd for $\mathrm{C}_{36} \mathrm{H}_{44} \mathrm{~N}_{2} \mathrm{O}_{6}$ : C 71.97, $\mathrm{H} 7.38, \mathrm{~N} 4.66$; Found $\mathrm{C}$ 71.68, H 7.23, N 4.71 .

\section{Results and discussion}

\subsection{Characterization of [pyridine- $\left.\mathrm{SO}_{3} \mathrm{H}\right] \mathrm{Cl}$}

The structure of the sulfonic acid functionalized pyridinium chloride was characterized on the basis of its ${ }^{1} \mathrm{H}$ and ${ }^{13} \mathrm{C}$ NMR, IR, UV, and MS data, which were presented in the Experimental section.

The IR spectrum of the catalyst contained a broad peak at 2650-3550 $\mathrm{cm}^{-1}$, which was attributed to the $\mathrm{O}-\mathrm{H}$ stretching of the $\mathrm{SO}_{3} \mathrm{H}$ group. In addition, the two peaks observed at 1042 and $1174 \mathrm{~cm}^{-1}$ were assigned to the vibrational modes of the $\mathrm{N}-\mathrm{SO}_{2}$ and $\mathrm{O}-\mathrm{SO}_{2}$ bonds (Fig. 1 ).

The ${ }^{1} \mathrm{H}$ and ${ }^{13} \mathrm{C}$ NMR spectra of the [pyridine- $\left.\mathrm{SO}_{3} \mathrm{H}\right] \mathrm{Cl}$ catalyst are shown in Fig. 2. The peak at $\delta=13.67$ in the ${ }^{1} \mathrm{H}$ NMR spectrum was assigned to the acidic hydrogen of the $\mathrm{SO}_{3} \mathrm{H}$ group. To confirm this assignment through a process of elimination, we also collected the ${ }^{1} \mathrm{H}$ NMR spectra of $\mathrm{ClSO}_{3} \mathrm{H}$ and pyridinium chloride in DMSO- $\mathrm{d}_{6}$ (the acidic hydrogens of pyridinium chloride and pyridinium chlorosulfonate gave similar chemical shifts). All of the samples involved in this ${ }^{1} \mathrm{H}$ NMR study were prepared at a concentration of $0.08 \mathrm{~mol} / \mathrm{L}$. The peaks corresponding to the acidic hydrogens of pyridinium chloride, $\mathrm{ClSO}_{3} \mathrm{H}$, and [pyridine- $\mathrm{SO}_{3} \mathrm{H}$ ] Cl were detected at $\delta=$ $11.37,13.45$, and 13.67 , respectively. The differences between

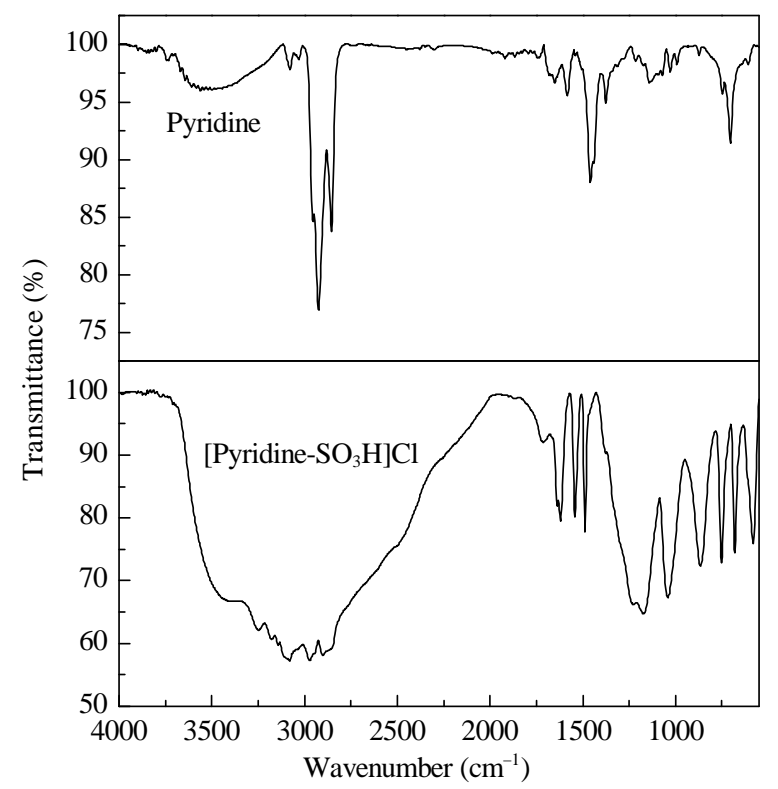

Fig. 1. IR spectra of sulfonic acid functionalized pyridinium chloride [pyridine- $\mathrm{SO}_{3} \mathrm{H}$ ]Cl. 

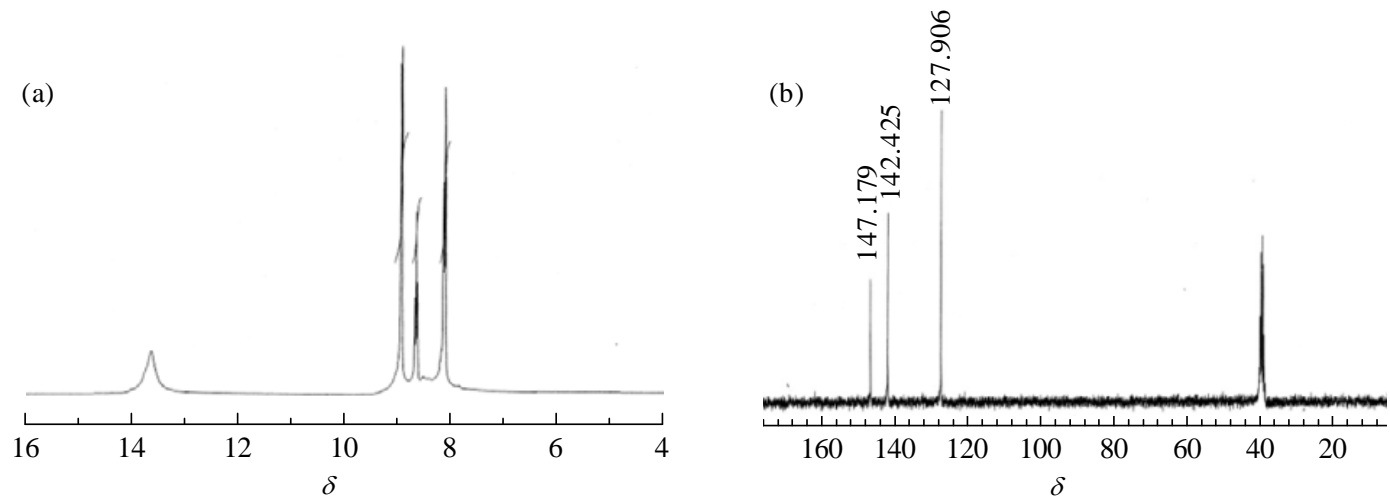

Fig. 2. ${ }^{1} \mathrm{H}(\mathrm{a})$ and ${ }^{13} \mathrm{C}(\mathrm{b}) \mathrm{NMR}$ spectra of sulfonic acid functionalized pyridinium chloride [pyridine- $\mathrm{SO}_{3} \mathrm{H}$ ]Cl.

the observed chemical shifts of the acidic hydrogen in these three species confirmed that the peak detected at $\delta=13.67$ had been correctly assigned to the $\mathrm{SO}_{3} \mathrm{H}$ group of the [pyridine- $\left.\mathrm{SO}_{3} \mathrm{H}\right] \mathrm{Cl}$ catalyst.

The UV/Vis absorbance spectrum of the catalyst was compared with those of pyridine and pyridinium chloride to highlight the differences between the UV/Vis absorbance pattern of the catalyst and pyridinium chloride. As shown in Fig. $3, \lambda_{\max }$ in the UV/Vis spectrum of the catalyst appeared at about $285 \mathrm{~nm}$. In the UV/Vis spectra of pyridine and pyridinium chloride, however, the $\lambda_{\max }$ values appeared at about 270 and $295 \mathrm{~nm}$, respectively (The compounds were dissolved in DMSO- $\mathrm{d}_{6}$ for UV studies at a concentration of $0.005 \mathrm{~mol} / \mathrm{L}$ ).

We also studied the formation of an N-S bond by exposing hydrogen chloride gas to sulfur trioxide pyridine complex as a white solid powder. This reaction also represents an alternative method for the preparation of [pyridine- $\mathrm{SO}_{3} \mathrm{H}$ ] Cl, where the white solid powder becomes a viscous colorless oil. The ${ }^{1} \mathrm{H}$ and ${ }^{13} \mathrm{C}$ NMR and IR spectra of the product were compared with those obtained from the other procedure described above and found to be identical. This result clearly proves that the structure of the [pyridine- $\mathrm{SO}_{3} \mathrm{H}$ ] Cl prepared in the current has been correctly characterized (Scheme 1).

Moreover, based on the results from the literature $[15,48,49]$, it is well known that sulfur trioxide pyridine complex $\left[\mathrm{C}_{5} \mathrm{H}_{5} \mathrm{~N}^{+} \mathrm{SO}_{3}{ }^{-}\right]$can be generated in dichloromethane at low

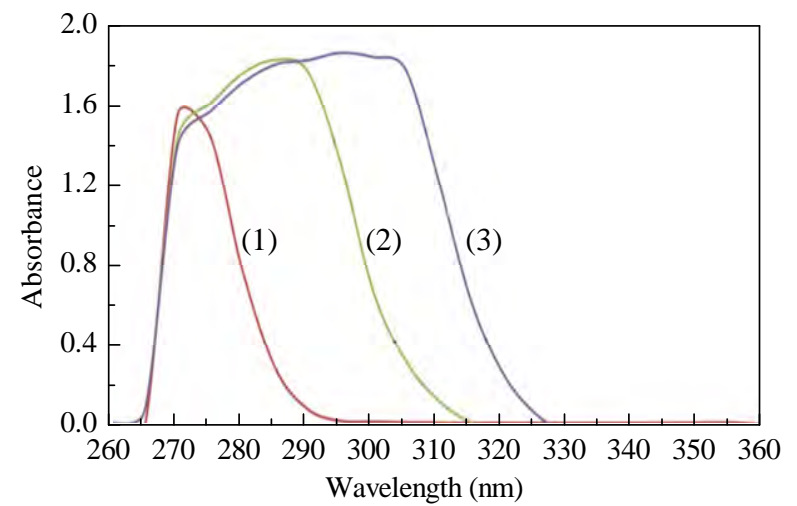

Fig. 3. UV spectra of pyridine (1), pyridinium chloride (2), and sulfonic acid functionalized pyridinium chloride [pyridine- $\left.\mathrm{SO}_{3} \mathrm{H}\right] \mathrm{Cl}(3)$ at room temperature in dimethyl sulfoxide. temperatures via the drop-wise addition of chlorosulfonic acid (1 equiv.) to pyridine (2 equiv.) (Scheme 3 ). Under these conditions, because pyridine is always present in excess compared with $\mathrm{ClSO}_{3} \mathrm{H}$, it is generally assumed that 1 equiv. of pyridine (as a nucleophile) attaches to the sulfur of $\mathrm{ClSO}_{3} \mathrm{H}$ to produce [pyridine- $\left.\mathrm{SO}_{3} \mathrm{H}\right] \mathrm{Cl}$, with another equiv. of pyridine functioning as a base to abstract the acidic hydrogen of [pyridine- $\mathrm{SO}_{3} \mathrm{H}$ ] Cl to yield $\mathrm{C}_{5} \mathrm{H}_{5} \mathrm{NHCl}$ and $\mathrm{C}_{5} \mathrm{H}_{5} \mathrm{~N}^{+} \mathrm{SO}_{3}$. In our current procedure for the synthesis of [pyridine- $\mathrm{SO}_{3} \mathrm{H}$ ] Cl, 1 equiv. of pyridine was added drop-wise to 1 equiv. of chlorosulfonic acid at low temperature. Under these conditions, there is always an excess of $\mathrm{ClSO}_{3} \mathrm{H}$ in the reaction mixture compared with pyridine. Thus, when pyridine reacts with $\mathrm{ClSO}_{3} \mathrm{H}$, [pyridine- $\left.\mathrm{SO}_{3} \mathrm{H}\right] \mathrm{Cl}$ would be formed and there would be no base present in the reaction mixture to abstract the acidic hydrogen to produce $\mathrm{C}_{5} \mathrm{H}_{5} \mathrm{~N}^{+} \mathrm{SO}_{3}{ }^{-}$ and $\mathrm{C}_{5} \mathrm{H}_{5} \mathrm{NHCl}$. Moreover, based on results from the literature $[15,48,49]$, the reaction of pyridine with chlorosulfonic acid at low temperature would occur via a nucleophilic substitution pathway (i.e., substitution of the $\mathrm{Cl}$ in $\mathrm{ClSO}_{3} \mathrm{H}$ by the nitrogen of pyridine) rather than the acid-base reaction (i.e., the abstraction of the hydrogen of $\mathrm{ClSO}_{3} \mathrm{H}$ by pyridine to afford $\left.\left[\mathrm{C}_{5} \mathrm{H}_{5} \mathrm{~N}-\mathrm{H}\right]\left[\mathrm{ClSO}_{3}\right]\right)$.

The sulfonic acid functionalized pyridinium chloride was also studied by TGA. The corresponding diagrams are shown in Fig. 4. TGA and DTGA of the catalyst showed that a mass loss occurred in one step. The [pyridine- $\mathrm{SO}_{3} \mathrm{H}$ ] Cl decomposed after $200{ }^{\circ} \mathrm{C}$ in one step.

\subsection{Catalytic activity of [pyridine $\left.-\mathrm{SO}_{3} \mathrm{H}\right] \mathrm{Cl}$}

Following on from our full characterization of [pyridine- $\left.\mathrm{SO}_{3} \mathrm{H}\right] \mathrm{Cl}$, we examined its catalytic activity towards the synthesis of hexahydroquinolines. The multi-component reaction of dimedone (2 $\mathrm{mmol}), 2$-methoxybenzaldehyde (2 $\mathrm{mmol}$ ),
2

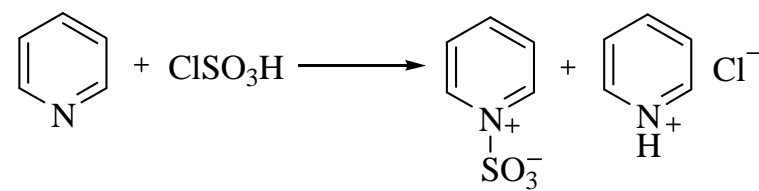

Scheme 3. Preparation of the sulfur trioxide pyridine complex $\left[\mathrm{C}_{5} \mathrm{H}_{5} \mathrm{~N}^{+} \mathrm{SO}_{3}^{-}\right]$by the reaction of pyridine with chlorosulfonic acid. 


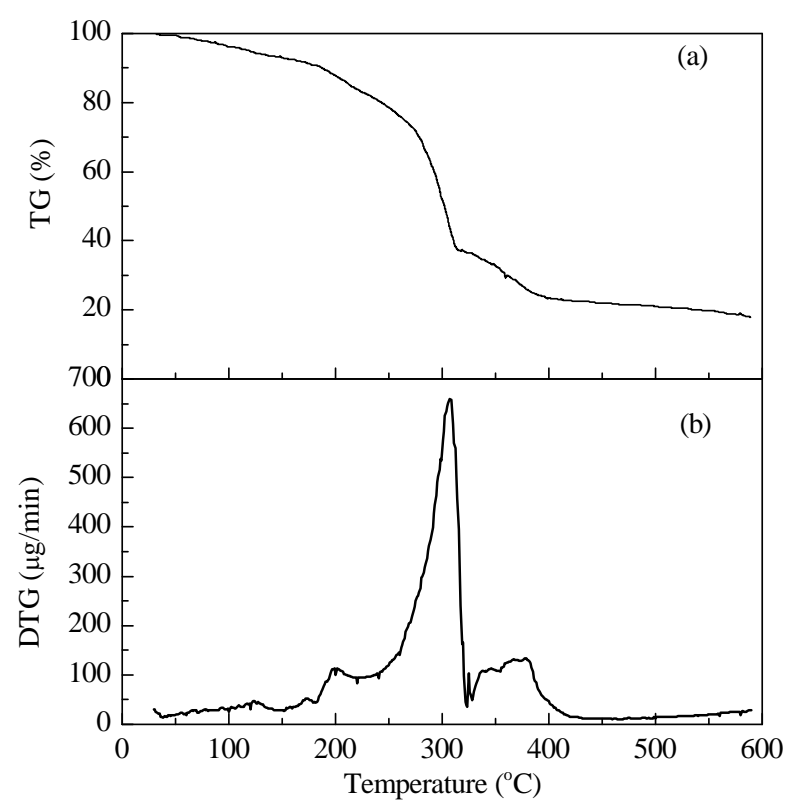

Fig. 4. TGA (a) and DTGA (b) diagrams of the [pyridine- $\mathrm{SO}_{3} \mathrm{H}$ ] Cl catalyst.

ethyl acetoacetate $(2 \mathrm{mmol})$, and ammonium acetate (2.4 mmol) was selected as a model reaction. The reaction was conducted in the presence of different amounts of [pyridine- $\left.\mathrm{SO}_{3} \mathrm{H}\right] \mathrm{Cl}$ at temperatures in the range of 25 to $90{ }^{\circ} \mathrm{C}$ in the absence of solvent. The results of these optimization experiments are summarized in Table 1 . It is clear from the results that a catalyst loading of $3 \mathrm{~mol} \%$ was sufficient to efficiently catalyze the reaction at $50{ }^{\circ} \mathrm{C}$, with the corresponding HHQ being obtained in $96 \%$ yield under these conditions within 11 min (Table 1 , entry 3 ).

The reaction of dimedone ( $2 \mathrm{mmol}), 2$-methoxybenzaldehyde ( $2 \mathrm{mmol})$, ethyl acetoacetate $(2 \mathrm{mmol})$, and ammonium acetate $(2.4 \mathrm{mmol})$ in the presence of [pyridine- $\left.\mathrm{SO}_{3} \mathrm{H}\right] \mathrm{Cl}$ was selected as a model reaction to compare the efficiency of the solution versus solvent-free conditions using a variety of different solvents at $50{ }^{\circ} \mathrm{C}$. All of the solvents tested provided low yields of the product, even after extended reaction time (Table 2).

To assess the general scope of the new catalyst, dimedone was reacted with a series of different aryl aldehydes (benzal-

\section{Table 1}

Effect of the [pyridine- $\left.\mathrm{SO}_{3} \mathrm{H}\right] \mathrm{Cl}$ catalyst amount and temperature on the reaction between dimedone, 2-methoxybenzaldehyde, ethyl acetoacetate, and ammonium acetate in the absence of solvent.

\begin{tabular}{lcccc}
\hline Entry & $\begin{array}{c}\text { Catalyst loading } \\
(\mathrm{mol} \%)\end{array}$ & $\begin{array}{c}\text { Temperature } \\
\left({ }^{\circ} \mathrm{C}\right)\end{array}$ & $\begin{array}{c}\text { Time } \\
(\mathrm{min})\end{array}$ & $\begin{array}{c}\text { Yield }^{\mathrm{a}} \\
(\%)\end{array}$ \\
\hline 1 & 1 & 50 & 25 & 61 \\
2 & 2 & 50 & 15 & 73 \\
3 & 3 & 50 & 11 & 96 \\
4 & 5 & 50 & 11 & 96 \\
5 & 3 & 25 & 15 & 78 \\
6 & 3 & 70 & 11 & 96 \\
7 & 3 & 90 & 11 & 96 \\
\hline
\end{tabular}

Table 2

Effect of different solvents on the reaction of dimedone, 2-methoxybenzaldehyde, ethyl acetoacetate, and ammonium acetate in the presence of [pyridine- $\mathrm{SO}_{3} \mathrm{H}$ ] Cl (1 mol\%).

\begin{tabular}{lcccc}
\hline Entry & Solvent & Temperature $\left({ }^{\circ} \mathrm{C}\right)$ & Time $(\mathrm{min})$ & Yield $^{\mathrm{a}}(\%)$ \\
\hline 1 & $\mathrm{CHCl}_{3}$ & 50 & 60 & 38 \\
2 & $\mathrm{EtOAc}$ & 50 & 60 & 45 \\
3 & EtOH & 50 & 60 & 41 \\
4 & hexane & 50 & 60 & 48 \\
5 & $\mathrm{CH}_{2} \mathrm{Cl}_{2}$ & reflux & 60 & 42 \\
6 & - & 50 & 11 & 96 \\
\hline a Isolated yield. & & &
\end{tabular}

dehyde as well as arylaldehyde possessing electron-withdrawing groups, electron-donating groups, and halogens), $\beta$-ketoesters (ethyl and methyl acetoacetate), and ammonium acetate under the optimized reaction conditions. The results are shown in Table 3. It is clear that all of reactions proceeded efficiently under the optimized conditions to afford the corresponding hexahydroquinolines in high yields over short reaction time. Thus, our new ionic liquid [pyridine- $\mathrm{SO}_{3} \mathrm{H}$ ] Cl is a highly efficient and general catalyst for the synthesis of HHQs.

It is important that ionic liquids and ionic liquids supported on solids possess good recyclability properties $[5,15,16]$. With this in mind, we assessed the recyclability of the [pyridine$\left.\mathrm{SO}_{3} \mathrm{H}\right] \mathrm{Cl}$ ionic liquid in terms of its application as a catalyst $(3$ mol\%) to the multi-component reaction of dimedone ( $2 \mathrm{mmol}$ ), 2-methoxybenzaldehyde (2 mmol), methyl acetoacetate $(2$ $\mathrm{mmol})$, and ammonium acetate $(2.4 \mathrm{mmol})$ at $50{ }^{\circ} \mathrm{C}$. Upon completion of the reaction, the reaction mixture was cooled to room temperature and diluted with ethyl acetate $(20 \mathrm{~mL})$. The resulting mixture was stirred under reflux for $3 \mathrm{~min}$ and then decanted (the product was soluble in hot ethyl acetate, whereas the catalyst was insoluble). The viscous oily residue was washed with hot ethyl acetate $(10 \mathrm{~mL})$ and dried to give the pure recycled catalyst, which was used for the next run of the reaction. The catalytic activity of [pyridine- $\mathrm{SO}_{3} \mathrm{H}$ ] Cl was restored to within the limits of the experimental error for four successive runs (see Table 4).

A mechanism has been proposed for the reaction as shown in Scheme 4. Thus, the conversion of dimedone to its enol form using [pyridine- $\mathrm{SO}_{3} \mathrm{H}$ ] Cl and its subsequent reaction with the activated aldehyde (activated by the catalyst) would give intermediate I. In contrast, the reaction of the activated $\beta$-ketoester (activated by the catalyst) with ammonia (resulted from ammonium acetate) would give enamine II. The reaction of intermediate I with enamine II would then afford intermediate III, which would be converted to IV by tautomerization. This intermediate would then undergo an intramolecular nucleophilic attack from the $\mathrm{NH}_{2}$ group to the activated carbonyl group with the loss of $\mathrm{H}_{2} \mathrm{O}$ to afford $\mathbf{V}$. The tautomerization of $\mathbf{V}$ would then give the observed hexahydroquinoline product. It is noteworthy that in the steps involving the activation of carbonyl groups through the donation of a proton from the [pyridine- $\left.\mathrm{SO}_{3} \mathrm{H}\right] \mathrm{Cl}$, the proton would be transferred back to the catalyst in a later step. This mechanism can therefore be confirmed by the literature $[39,41,43]$. 
Table 3

The solvent-free synthesis of HHQs from dimedone, arylaldehydes, $\beta$-ketoesters, and ammonium acetate catalyzed by $\left[\mathrm{pyridine-} \mathrm{SO}_{3} \mathrm{H}\right] \mathrm{Cl}$ at $50{ }^{\circ} \mathrm{C}$.

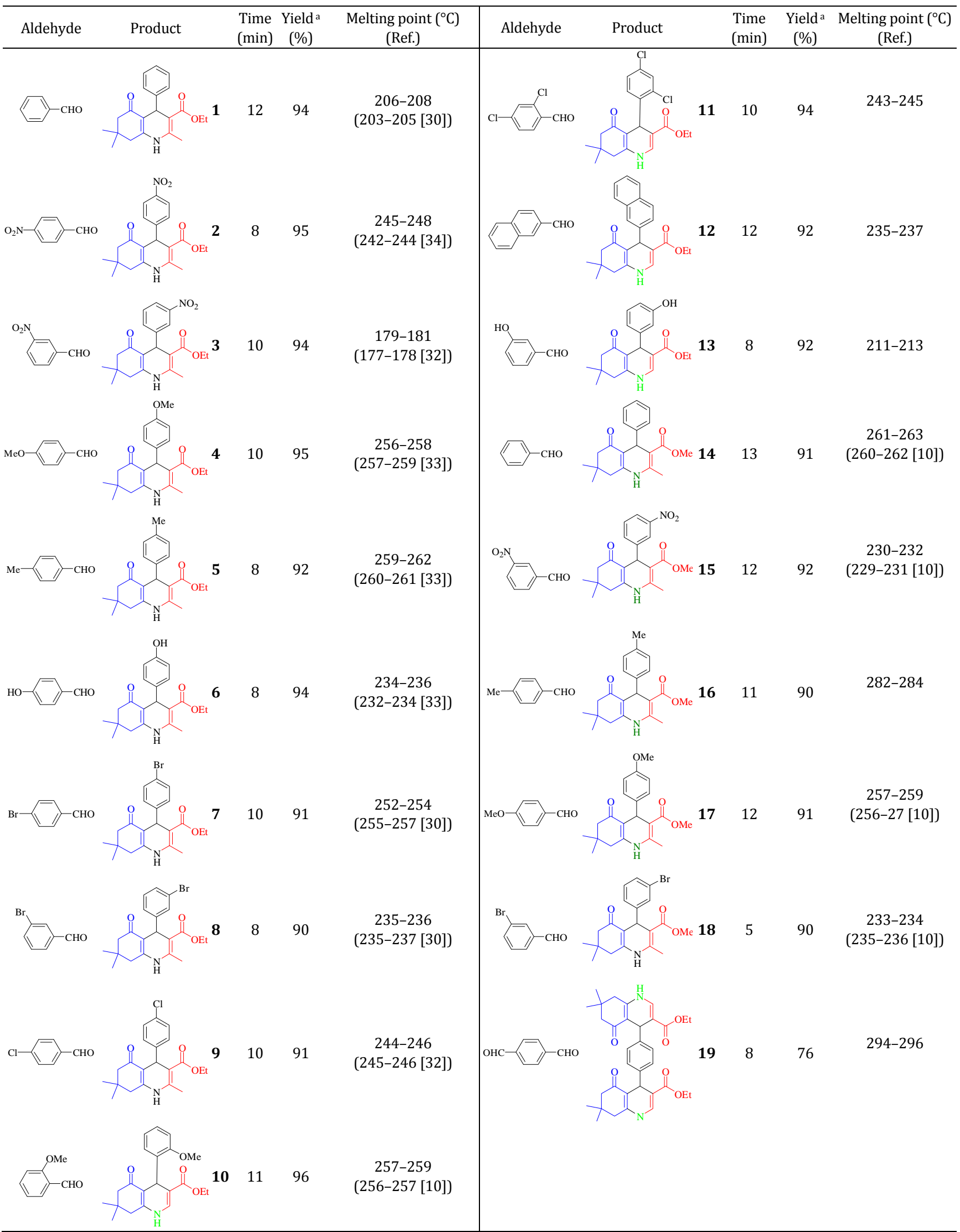


Table 4

The condensation of dimedone with 2-methoxybenzaldehyde, ethyl acetoacetate, and ammonium acetate using recycled [pyridine- $\left.\mathrm{SO}_{3} \mathrm{H}\right] \mathrm{Cl}$.

\begin{tabular}{lcc}
\hline Run & Time (min) & Yield ${ }^{a}(\%)$ \\
\hline 1 & 96 & 11 \\
2 & 93 & 13 \\
3 & 90 & 10 \\
4 & 85 & 15 \\
\hline
\end{tabular}

a Yield of purified product.

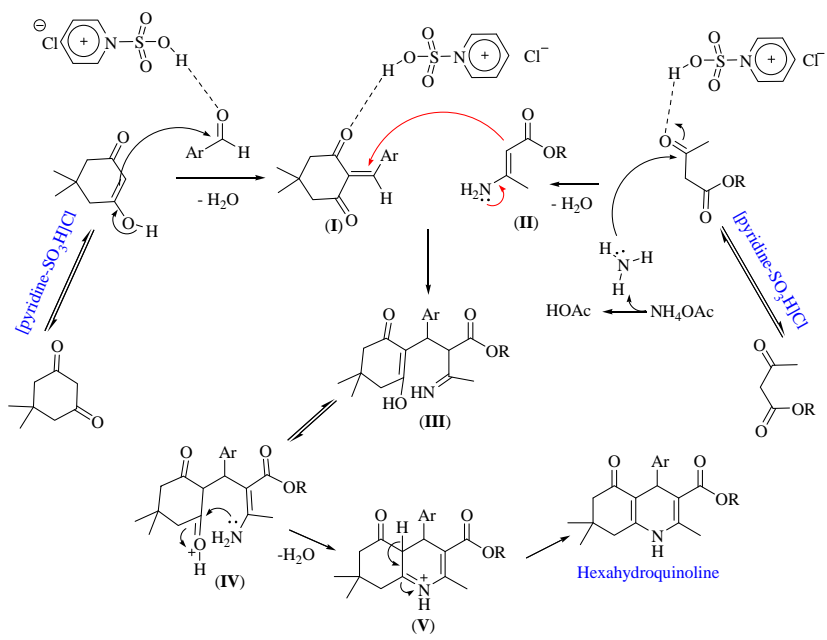

Scheme 4. Proposed mechanism for the synthesis of hexahydroquinolines promoted by [pyridine- $\left.\mathrm{SO}_{3} \mathrm{H}\right] \mathrm{Cl}$.

\section{Conclusions}

We have introduced the Brönsted acidic ionic liquid [pyridine- $\left.\mathrm{SO}_{3} \mathrm{H}\right] \mathrm{Cl}$ as a novel, highly efficient, general, and homogeneous catalyst for the one-pot multi-component reaction of dimedone, aromatic aldehydes, $\beta$-ketoesters, and ammonium acetate leading to hexahydroquinolines. The advantages of the current protocol include its efficiency, broad scope, high product yields, short reaction time, clean reaction profile, simplicity, low cost, ease of preparation, and the recyclability of the catalyst.

\section{Acknowledgements}

The authors gratefully acknowledge partial support for this work from the Research Affairs Office of Sayyed Jamaleddin Asadabadi University, and Bu-Ali Sina University (Grant no. 32-1716 entitled development of chemical methods, reagents and molecules), and the Center of Excellence in Development of Chemical Method (CEDCM), Hamedan, I.R. Iran.

\section{References}

[1] Pavlinac J, Zupan M, Laali K K, Stavber S. Tetrahedron, 2009, 65: 5625

[2] Olivier-Bourbigou H, Magna L, Morvan D. Appl Catal A, 2010, 373: 1

[3] Hapiot P, Lagrost C. Chem Rev, 2008, 108: 2238

[4] Wasserscheid P, Welton T. Ionic Liquids in Synthesis. Weinheim:
Wiley-VCH, 2008

[5] Hasaninejad A, Zare A, Shekouhy M, Ameri Rad J. J Comb Chem, 2010, 12: 844

[6] Zare A, Parhami A, Moosavi-Zare A R, Hasaninejad A, Khalafi-Nezhad A, Beyzavi M H. Can J Chem, 2009, 87: 416

[7] Eichmann M, Keim W, Haumann M, Melcher B U, Wasserscheid P.J Mol Catal A, 2009, 314: 42

[8] Kim Y J, Varma R S. Tetrahedron Lett, 2005, 46: 1467

[9] Öchsner E, Schneider M J, Meyer C, Haumann M, Wasserscheid P. Appl Catal A, 2011, 399: 35

[10] Zare A, Abi F, Moosavi-Zare A R, Beyzavi M H, Zolfigol M A. J Mol Liq, 2013, 178: 113

[11] Shaterian H R, Ranjbar M.J Mol Liq, 2011, 160: 40

[12] Zolfigol M A, Khakyzadeh V, Moosavi-Zare A R, Zare A, Azimi S B, Asgari Z, Hasaninejad A. C R Chim, 2012, 15: 719

[13] Zolfigol M A, Khazaei A, Moosavi-Zare A R, Zare A.J Iran Chem Soc, 2010, 7: 646

[14] Shaterian H R, Ranjbar M, Azizi K. J Mol Liq, 2011, 162: 95

[15] Zare A, Moosavi-Zare A R, Merajoddin M, Zolfigol M A, Hekmat-Zadeh T, Hasaninejad A, Khazaei A, Mokhlesi M, Khakyzadeh V, Derakhshan-Panah F, Beyzavi M H, Rostami E, Arghoon A, Roohandeh R.J Mol Liq, 2012, 167: 69

[16] Zolfigol M A, Khazaei A, Moosavi-Zare A R, Zare A, Khakyzadeh V. Appl Catal A, 2011, 400: 70

[17] Zolfigol M A, Khazaei A, Moosavi-Zare A R, Zare A. Org Prep Proced Int, 2010, 42: 95

[18] Zolfigol M A, Khazaei A, Moosavi-Zare A R, Zare A, Kruger H G, Asgari Z, Khakyzadeh V, Kazem-Rostami M. J Org Chem, 2012, 77: 3640

[19] Zhu J, Bienayme H. Multicomponent Reactions. Weinheim: Wiley, 2005

[20] Khazaei A, Zolfigol M A, Moosavi-Zare A R, Zare A, Khojasteh M, Asgari Z, Khakyzadeh V, Khalafi-Nezhad A. Catal Commun, 2012, 20: 54

[21] Khazaei A, Zolfigol M A, Moosavi-Zare A R, Zare A, Parhami A, Khalafi-Nezhad A. Appl Catal A, 2010, 386: 179

[22] Glass B D, Brown M E, Drummond P M. In: Albini A, Fasani E eds. Drugs: Photochemistry and Photostability. Cambridge: The Royal Society of Chemistry, 1998. 134

[23] Beijersbergen van Henegouwen G M J. In: Testa B, Meyer U A eds. Advances in Drug Research. Vol 29. London: Academic Press, 1997. 79

[24] [24] Görlitzer K, Baltrusch H J, Gossnitzer E, Wendelin W. Pharmazie, 2000, 55: 35

[25] De Vries H, Beijersbergen van Henegouwen G M J. Photochem Photobiol, 1995, 62: 959

[26] Eisner U, Kuthan J. Chem Rev, 1972, 72: 1

[27] Stout D M, Meyers A I. Chem Rev, 1982, 82: 223

[28] Chorvat R J, Rorig K J. J Org Chem, 1988, 53: 5779

[29] Kappe C O, Fabian W M F, Semones M A. Tetrahedron, 1997, 53: 2803

[30] Kappe C 0. Tetrahedron, 1993, 49: 6937

[31] Safak C, Sahin I, Sunal R. Arzneimittel Forsch, 1990, 40: 119

[32] Simsek R, Ismailoglu U B, Safak C, Sahin-Erdemli I. Farmaco, 2000, 55: 665

[33] Larsen R D, Corley E G, King A O, Carrol J D, Davis P, Verhoeven T R, Reider P J, Labelle M, Gauthier J Y, Xiang Y B, Zamboni R J. J Org Chem, 1996, 61: 3398

[34] Chen Y L, Fang K C, Sheu J Y, Hsu S L, Tzeng C C. J Med Chem, 2001, 44: 2374

[35] Roma G, Di Braccio M, Grossi G, Mattioli F, Chia M. Eur J Med Chem, 2000, 35: 1021 


\section{Graphical Abstract}

Chin. J. Catal., 2013, 34: 1936-1944 doi: 10.1016/S1872-2067(12)60678-0

Synthesis of hexahydroquinolines using the new ionic liquid sulfonic acid functionalized pyridinium chloride as a catalyst

Ardeshir Khazaei*, Mohammad Ali Zolfigol*, Ahmad Reza Moosavi-Zare*, Javad Afsar, Abdolkarim Zare, Vahid Khakyzadeh, Mohammad Hassan Beyzavi

Bu-Ali Sina University, Iran; University of Sayyed Jamaleddin Asadabadi, Iran; Payame Noor University, Iran;

Freie Universität Berlin, Germany

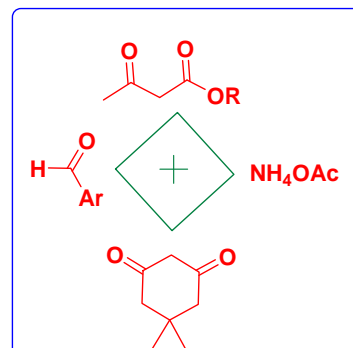

$\mathrm{R}=\mathrm{Me}, \mathrm{Et}$

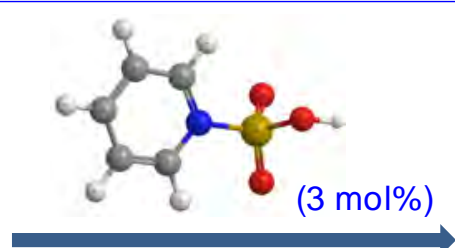

Solvent-free $50^{\circ} \mathrm{C}$<smiles>[R]OC(=O)C1=C(C)NC2=C(C(=O)CC(C)(C)C2)C1Br</smiles>

$1-19$

Sulfonic acid functionalized pyridinium chloride [pyridine- $\mathrm{SO}_{3} \mathrm{H}$ ]Cl has been prepared as a novel Brönsted acidic ionic liquid and characterized using a range of analytical techniques. This compound efficiently catalyzed the synthesis of hexahydroquinolines at $50{ }^{\circ} \mathrm{C}$ under solvent-free conditions.

[36] Geng X, Li S S, Bian X Q, Xie Z Y, Wang C D. ARKIVOC, 2008: 50

[37] Maguire M P, Sheets K R, McVety K, Spada A P, Zilberstein A. J Med Chem, 1994, 37: 2129

[38] Billker O, Lindo V, Panico M, Etienne A E, Paxton T, Dell A, Rogers M, Sinden R E, Morris H R. Nature, 1998, 392: 289

[39] Song S J, Shan Z X, Jin Y. Synth Commun, 2010, 40: 3067

[40] Zhang X L, Sheng S R, Liu X L, Liu XL. ARKIVOC, 2007: 79

[41] Kumar A, Maurya R A. Tetrahedron, 2007, 63: 1946

[42] Wang L M, Sheng J, Zhang L, Han J W, Fan Z Y, Tian H, Qian C T. Tetrahedron, 2005, 61:1539
[43] Ziarani G M, Badiei A R, Khaniania Y, Haddadpour M. Iran J Chem Chem Eng, 2010, 29: 1

[44] Pasunooti K K, Jensen C N, Chai H, Leow M L, Zhang D W, Liu X W.J Comb Chem, 2010, 12: 577

[45] Surasani R, Kalita D, Rao AV D, Yarbagi K, Chandrasekhar K B. J Fluorine Chem, 2012, 135: 91

[46] Hong M, Cai C, Yi W B.J Fluorine Chem, 2010, 131: 111

[47] Saha M, Pal A K. Tetrahedron Lett, 2011, 52: 4872

[48] Ajinomoto Co Inc. JP Patent 59134751 A. 1984

[49] Sakota N, Nomura S, Ito S. JP Patent 03024039 A. 1991 